\title{
Quasi-phase-matched high harmonic generation in gas-filled photonic crystal fibers
}

\author{
Patrick N. Anderson ${ }^{1, *}$, Florian Wiegandt ${ }^{1}$, Fei Yu ${ }^{2}$, Daniel J. Treacher ${ }^{1}$, David T. Lloyd ${ }^{1}$, \\ Peter J. Mosley ${ }^{2}$, Jonathan C. Knight ${ }^{2}$, Simon M. Hooker ${ }^{1}$, Ian A. Walmsley ${ }^{1}$ \\ ${ }^{I}$ Clarendon Laboratory, University of Oxford, Parks Road, Oxford, OXI 3PU, UK \\ ${ }^{2}$ Centre for Photonics and Photonic Materials, Department of Physics, University of Bath, Bath, BA2 7AY, UK \\ *patrick.anderson@physics.ox.ac.uk
}

\begin{abstract}
We investigate HHG in gas-filled PCFs with microjoule driving lasers. QPM is implemented for the first time, enhancing the narrow-band flux at $30 \mathrm{eV}$ by a factor of 60 .

OCIS codes: (140.7240) UV, EUV, and X-ray lasers; (060.5295) Photonic crystal fibers
\end{abstract}

\section{Introduction}

Driving efficient high harmonic generation (HHG) with few- $\mu \mathrm{J}$ fs laser pulses is a critical step on the roadmap to realizing compact, high average power sources of coherent extreme ultraviolet (EUV) radiation for lithography and imaging applications. To reach a sufficiently strong E-field to perform HHG these pulses must be focused tightly, and as a result the interaction volume is greatly reduced. One seemingly elegant solution to this problem can be borrowed from the $\mathrm{mJ}$ regime where HHG is often restricted to a gas-filled capillary of radius $a$ [1]. The $1 / a^{3}$ scaling of capillary losses can be mitigated by using photonic crystal fibers (PCFs) [2], but the $1 / a^{2}$ dependence of the propagation constant cannot. As reabsorption is strong throughout much of the EUV, correcting for this using multiatmosphere pressures [3] is not advantageous and quasi-phase-matching (QPM) is the preferred approach. Here we demonstrate QPM of HHG for the first time within a PCF, enhancing the narrow-band flux of the $19^{\text {th }}$ harmonic (30 $\mathrm{eV})$ of a Ti:sapphire laser by a factor of 60 .

\section{Concept}

We have developed a variation of multimode-QPM (MM-QPM) [4] applicable to PCFs. Our new scheme is illustrated by the electromagnetic simulation in Fig. 1(a). Here, a "control" pulse is coupled into a PCF such that several higher-order modes are excited. As the field propagates waveguide dispersion causes these modes to walkoff, isolating them in time. At some later time a second "drive" pulse is injected into the fiber and the relative delay adjusted so its fundamental mode overlaps with a higher-order mode of the control pulse (here an $\mathrm{EH}_{1,4}$-like mode) towards the exit of the fiber. Once their envelopes are overlapped the carrier waves can beat, causing the drive pulse to undergo rapid amplitude modulation as shown in Fig. 1(b). By varying the arrival time of the drive pulse and the coupling of the control pulse the QPM period and modulation depth can be adjusted to better match the desired harmonic.
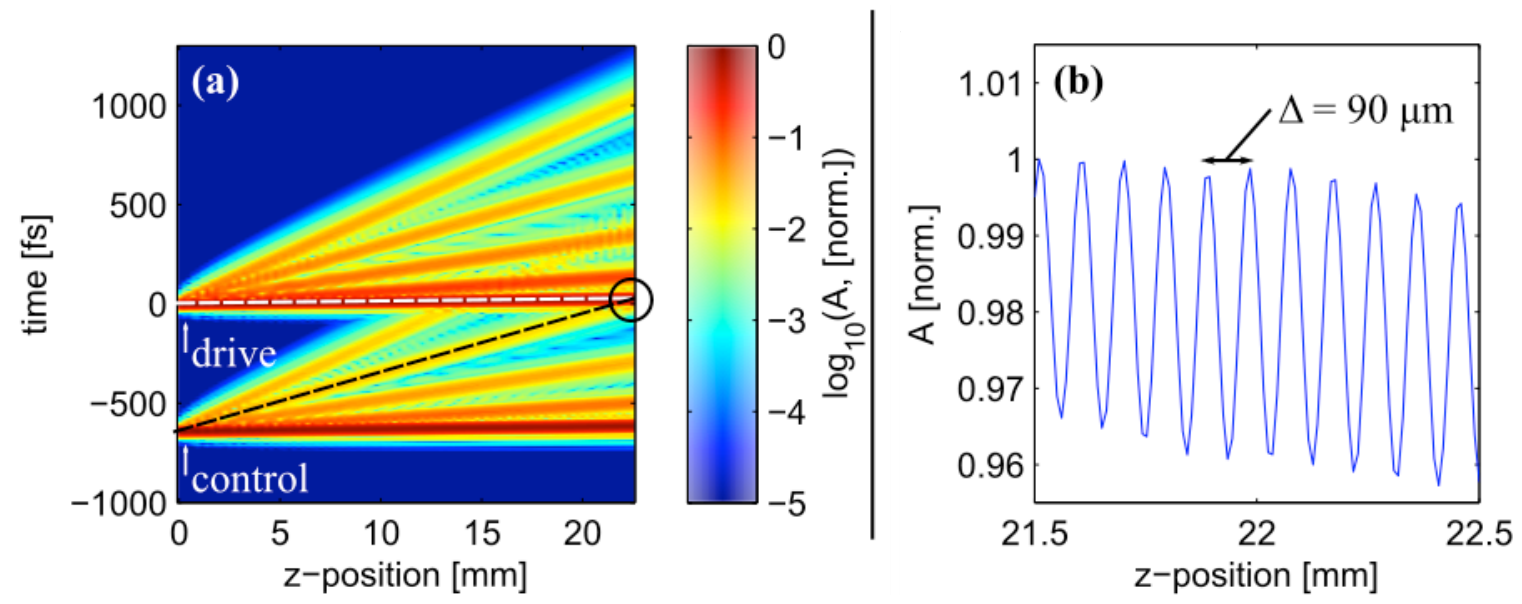

Fig. 1. Proposed QPM mechanism. (a) Simulated on-axis E-field amplitudes for control and drive pulses propagating through a gas-filled PCF with $a=11.25 \mu \mathrm{m}$. The relative delay of the two pulses is adjusted such that the fundamental mode of the drive pulse overlaps with a higherorder mode of the control pulse towards the exit of the fiber. The beating between these modes produces the z-dependent amplitude modulation required for QPM. In this particular example the modulation period is 90 um with a modulation depth of 0.03 (b). 
(a)

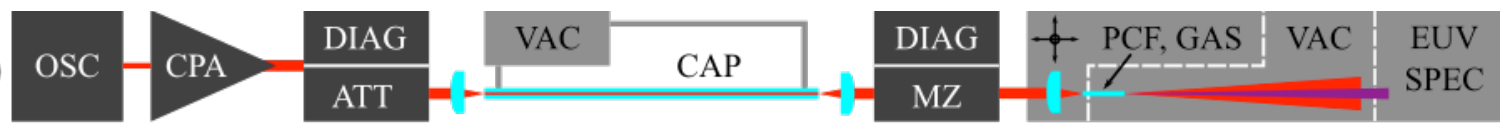
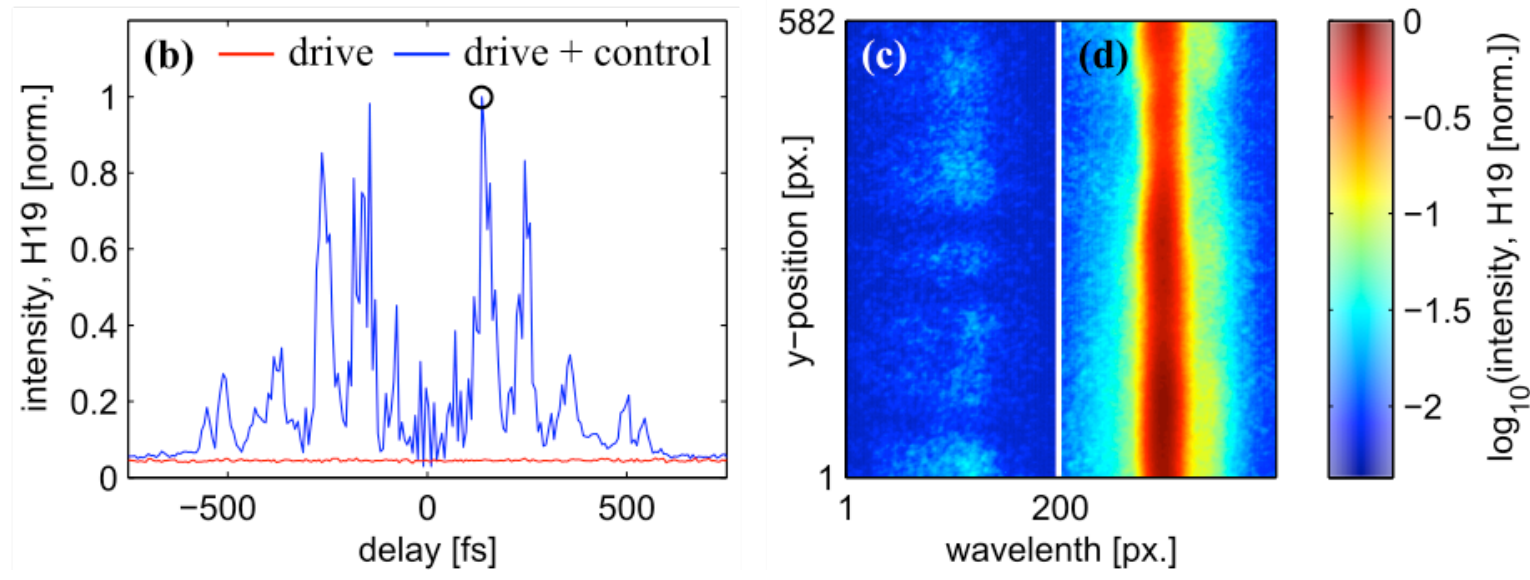

Fig. 2. (a) Schematic of the experimental beamline: OSC, oscillator; CPA, chirped-pulse amplifier; DIAG, diagnostic instrumentation; ATT, variable attenuator; VAC, vacuum; CAP, capillary-type fiber; MZ, Mach-Zehnder interferometer; PCF, photonic crystal fiber; GAS, gas supply; EUV SPEC, EUV spectrometer. (b) The intensity of H19 measured as a function of drive pulse delay. When the control pulse is introduced (blue) significant enhancements in flux are observed along with a periodic structure consistent with MM-QPM. The spatio-spectral distribution at the highlighted delay (black circle) is plotted with (d) and without (c) the control pulse. From this the narrow-band enhancement attributed to MMQPM is estimated to be 60 at $30 \mathrm{eV}$.

\section{Experimental results}

The experimental beamline is shown in Fig. 2(a). A Ti:sapphire based laser system producing $30 \mathrm{fs}$ pulses at $1 \mathrm{KHz}$ is attenuated, spatially filtered (within an evacuated capillary) and fed into a Mach-Zehnder interferometer to give identical time-delayed control and drive pulses. These are coupled into a $22.5 \mathrm{~mm}$ section of $a=11.25 \mu \mathrm{m}$ hollow core negative curvature PCF [5] spanning two vacuum chambers by translating a lens. The first chamber is filled with 800 mbar of argon and high vacuum is maintained throughout the second chamber and EUV spectrometer. With only the drive pulse present odd harmonics between $(\mathrm{H}) 15-23$ are detected at a threshold of approximately 5 $\mu \mathrm{J}$. As anticipated the signal is very weak (Fig, 2(c)), however at higher pulse energies the harmonics undergo significant broadening suggesting an ionization-induced distortion of the drive pulse. Fig. 2(b) shows the integrated intensity of $\mathrm{H} 19(30 \mathrm{eV})$ as a function of delay when both pulses are present and attenuated to $5 \mu \mathrm{J}$. The small enhancement close to $t=0$ fs is caused by harmonic broadening when drive and control pulses overlap coherently. Away from this region the periodic structure is consistent with MM-QPM. An additional signature of QPM is given in Fig. 2(c-d) where the enhancement is shown to be narrow-band. Finally, the narrow-band enhancement in flux attributed to MM-QPM can be quantified by a factor of 60 at $30 \mathrm{eV}$.

\section{References}

[1] A. Rundquist, C. G. Durfee, Z. Chang, C. Herne, S. Backus, M. M. Murnane and H. C. Kapteyn, "Phase-matched generation of coherent soft X-rays," Science 280, 1412-1415 (1998).

[2] O. H. Heckl, C. R. E. Baer, C. Kränkel, S. V. Marchese, F. Schapper, M. Holler, T. Südmeyer, J. S. Robinson, J. W. G. Tisch, F. Couny, P. Light, F. Benabid and U. Keller, "High harmonic generation in a gas-filled hollow-core photonic crystal fiber" Appl. Phys. B 97, 369-373 (2009).

[3] T. Popmintchev, M. Chen, D. Popmintchev, P. Arpin, S. Brown, S. Ališauskas, G. Andriukaitis, T. Balčiunas, O. Mücke, A. Pugzlys, A. Baltuška, B. Shim, S. E. Schrauth, A. Gaeta, C. Hernández-García, L. Plaja, A. Becker, A. Jaron-Becker, M. M. Murnane and H. C. Kapteyn, "Bright Coherent Ultrahigh Harmonics in the keV X-ray Regime from Mid-Infrared Femtosecond Lasers," Science 336, 1287-1291 (2012).

[4] M. Zepf, B. Dromey, M. Landreman, P. Foster and S. M. Hooker, "Bright Quasi-Phase-Matched Soft-X-Ray Harmonic Radiation from Argon Ions” Phys. Rev. Lett. 99, 143901 (2007).

[5] F. Yu and J. C. Knight, "Spectral attenuation limits of silica hollow core negative curvature fiber" Opt. Express 21, 21466-21471 (2013). 